\title{
Leben nach dem Infarkt
}

\section{Initiative Herzbewusst fördert gezielte Nachsorge}

\author{
Nach einem überstandenen Herz- \\ infarkt besteht weiterhin ein hohes \\ Risiko für die Patienten. Diese auf \\ ihrem Weg in das Leben nach dem \\ Infarkt zu begleiten, ist das Ziel der \\ „Initiative Herzbewusst“.
}

Etwa 250000 Bundesbürger erleiden jährlich einen Myokardinfarkt. Jene, die ihn überleben, sind keineswegs außer Gefahr. „Einmal Herzpatient, immer Herzpatient“, so Dr. Franz Goss, München. Das bestätigt auch eine aktuelle Auswertung des Deutschen Herzregisters: Einer von acht $\mathrm{Pa}$ tienten überlebt das erstes Jahr nach seinem Infarkt nicht.

Das gibt zu denken angesichts dessen, dass die akute Versorgung von Herzinfarktpatienten in Deutschland laut Goss sehr gut ist. Was fehlt, ist „,die gezielte Nachsor- ge“. Diese Lücke zu schließen, ist das Anliegen der „Initiative Herzbewusst - Leben nach dem Infarkt". Der Startschuss der bundesweiten Aufklärungs- und Informationskampagne fiel Mitte November 2010. Erklärte Ziele der Initiatoren (Bundesverband Niedergelassener Kardiologen e.V. und AstraZeneca) sind die Vermeidung eines erneuten akuten Koronarsyndroms und die Verbesserung der langfristigen Versorgung der Betroffenen.

Nach den Worten von Goss, ,wollen und müssen wir die Patienten dazu bewegen, selbst aktiv zur Senkung ihres Langzeitrisikos beizutragen“. Dafür sind die konsequente Einnahme der verordneten Medikamente und insbesondere ein aktiver Lebensstil erforderlich. Wie u.a. die Metaanalyse der Cochrane-Datenbank zeigte, senkt ein regelmäßiges körperliches Training die kardiovaskuläre Mortalität um 32\%. Um die Sensibilität und das Bewusstsein für eine intensive Nachsorge nach dem Infarkt zu fördern, hält die Initiative ein umfangreiches Informationsangebot für Patienten und Vertreter aus dem Gesundheitswesen bereit. Neben einem Herzrisiko-Check und einem Herzinfarkt-Pass stellt die Initiative auf ihrer Website www.herzbewusst.de umfassende Informationen rund um das akute Koronarsyndrom zur Verfügung. Für 2011 sind Weiterbildungsveranstaltungen für Fachkreise geplant und im April des Jahres wird der „Herzbewusst-Herztag“ stattfinden.

(bfro) II

II Pressekonferenz, Hamburg, 16. November 2010 (Veranstalter: AstraZeneca $\mathrm{GmbH}$, Bundesverband Niedergelassener Kardiologen e.V.)

\section{Multidimensionale Lipidtherapie Nikotinsäure richtig dosieren - und nicht ohne Statin}

\begin{abstract}
Studien, in denen die Wirkung einer lipidsenkenden Therapie auf atherosklerotische Plaques untersucht wurde, zeigen, dass die Atherosklerose vollständig zum Stillstand bzw. sogar zurückgebildet werden kann. Dies gelingt ab einem LDL/HDL-Quotienten unter 1.
\end{abstract}

Nur bei wenigen Patienten kann dieser Quotient allein mit einer Statintherapie erreicht werden. In den meisten Fällen ist dazu ein multidimensionales Lipidmanagement notwendig, welches das atherogene Potenzial sowohl von LDL-Cholesterin als auch von HDL-Cholesterin, den Triglyzeriden und des Lipoproteins a reduziert. Dies gelingt am besten durch die Kombination eines Statins mit Nikotinsäure.

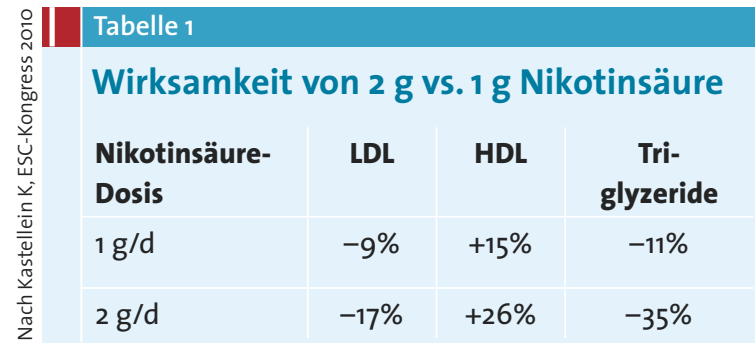

Flush-Phänomen. Dieses ist deutlich seltener geworden, seit retardierte Nikotinsäure zusammen mit Laropiprant (als Tredaptive ${ }^{\circledR}$ ) verabreicht wird. Laropiprant blockert den Rezeptor in der Haut, über den der Flush ausgelöst wird. „Der Unterschied zwischen Nikotinsäure und Nikotinsäure plus

Allerdings muss die Nikotinsäure dazu in der vollen Dosis von $2 \mathrm{~g}$ gegeben werden, betonte Prof. John Kastellein, Amsterdam. Wer aus Angst vor Nebenwirkungen Nikotinsäure nur mit $1 \mathrm{~g}$ dosiert bzw. auf die Dosisverdopplung nach vier Wochen verzichtet, verschenkt einen Großteil der Wirksamkeit (Tab. 1). Die am meisten störende Nebenwirkung der Nikotinsäure ist das
Laropiprant entspricht etwa einem Flush pro Tag im Vergleich zu einem Flush pro Woche", veranschaulichte Kastellein. Er rät dazu, mit den Patienten diese Nebenwirkung zu besprechen und sie zu bitten, diese vor allem in den ersten sechs bis acht Therapiewochen zu ertragen. Nach der Neueinstellung und Dosisverdopplung lässt sie deutlich nach. Auf lange Sicht 